\title{
Independent, Tough Identical Results: The Class of Tweedie on Power Variance Functions and the Class of Bar-Lev and Enis on Reproducible Natural Exponential Families
}

\author{
Shaul K. Bar-Lev \\ Correspondence: Shaul K. Bar-Lev, Holon Institute of Technology, Israel; University of Haifa, Haifa, Israel. E-mail: \\ shaulb@hit.ac.il;barlev@stat.haifa.ac.il
}

\author{
Received: November 13, 2019 Accepted: December 24, 2019 Online Published: December 26, 2019 \\ doi:10.5539/ijsp.v9n1p30 \\ URL: https://doi.org/10.5539/ijsp.v9n1p30
}

\begin{abstract}
The Rao-Blackwell theorem has had a fundamental role in statistical theory. However, as opposed to what seems natural, Rao and Blackwell did not investigate and write the theorem jointly. In fact, they both published the same result independently, two years apart. Indeed, as C.R. Rao writes in Wikipedia: "the result on one parameter case was published by Rao (1945) in the Bulletin of the Calcutta Mathematical Society and by Blackwell (1947) in The Annals of Mathematical Statistics. Only Lehmann and Scheffé (1950) called the result as Rao-Blackwell theorem”. Forty years later, a situation very similar to the previous one seems to have happened. Tweedie (1984) in a paper published in a proceedings to a conference held in Calcutta and Bar-Lev and Enis (1986) in a paper published in The Annals of Statistics both presented for the first time, albeit two years apart, independently and in different contexts, the class of natural exponential families having power variance functions (NEF-PVFs). Tweedie's results were then mentioned by Jorgensen (1987) in his fundamental paper on exponential dispersion models published in the Journal of the Royal Statistical Society, Series B. Jorgensen, however, mentioned also other researchers, including Bar-Lev and Enis, as dealt with the same problem. Nonetheless, Jorgensen (1987) stated in his paper that "The most complete study" of NEF-PVFs was given by Tweedie (1984), a statement which has led to naming the class of NEF-PVFs as the Tweedie class. This statement of Jorgensen is entirely and utterly incorrect. Accordingly, one of the goals of this note is to 'prove' such incorrectness. Based on this 'proof' it will be evident, so I trust, that both Bar-Lev and Enis should have received the appropriate credit by re-naming the class of NEF-PVFs via the exploitation of the names of Tweedie, Bar-Lev and Enis. This would resemble the dignified and elegant manner Lehmann and Scheffé acted on the Rao-Blackwell Theorem. Notwithstanding, the main aim of the note is to encourage young researchers to present their results with self-confidence and to get the credit they deserve.
\end{abstract}

Keywords: natural exponential family, power variance function, Rao-Blackwell theorem, reproducibility, Tweedie class

\section{Background and Main Results}

This note has a two-fold goal, the first is pedagogical, and the second is partially related to perhaps some straitlaced issues as well as inattention and inadequacy of details to the facts. I believe that the discussion of the issues I will present is of great importance to both graduate students and researchers (and especially the youngest among them). The topics of discussion are related to how the Rao-Blackwell theorem was first presented in the literature during the late 1940s compared to the way Tweedie's results (Tweedie (1984)) on the class of natural exponential families having power variance functions (NEF-PVFs) were presented by Jorgensen (1987) in his fundamental paper on exponential dispersion models. Since Jorgensen's presentation, the class of NEF-PVFs is known in the literature as the Tweedie class which is implemented in a wide variety of disciplines for data modeling.

For this comparison, I have carefully examined all relevant details and facts related to these two issues. I will first start with the Rao-Blackwell theorem issue. I remember the period when I was a graduate student and became familiar with the Rao-Blackwell theorem. I knew then to prove it at various mathematical levels of proof. As the theorem appears in a large number of textbooks on various levels, and of course, in journal articles, I was entirely confident in that period that Rao and Blackwell jointly investigated the problem and then published their results in a journal's paper that appeared in the late 1940s. I was wrong, and only towards the end of my doctoral studies at the Technion (Israel Institute of Technology) in 1981 did I discover the 'pure' truth. The two did not write the paper jointly. In fact, they both published the same result independently, two years apart. Indeed, as Dr. Calyampudi Radhakrishna Rao writes in Wikipedia: "the result on one parameter case was published by Rao (1945) in the Bulletin of the Calcutta Mathematical Society and by Blackwell (1947) in The Annals of Mathematical Statistics. Lehmann and Scheffé (1950) called the result as Rao-Blackwell theorem. In computational terminology, it is called Rao-Blackwellized Filter". So, actually, the two renowned scientists, Lehmann 
and Scheffé, acted in a dignified and logical way by naming the theorem the Rao-Blackwell theorem.

It is almost superfluous to say that the difference between the two years of publication of the two results is entirely reasonable, especially since the Rao's result was published in an Indian bulletin which, according to any reasonable assumption, was almost negligible those days in western university libraries. This adds to the fact that search engines in those years were only dreams of the sort that appear in Jules Verne's books.

Now naturally arises the following question: Why do I raise such a well-known issue again? The answer to this is rather simple and related to a very similar situation, albeit with a considerable difference of years. Such a similar situation is related to Tweedie's paper published in 1984 in the Proceedings of the Indian Institute Golden Jubilee International Conference and to the Bar-Lev and Enis' paper published in 1986 in The Annals of Statistics. Both Tweedie and Bar-Lev and Enis discovered and presented for the first time, albeit two years apart, independently and in different contexts, the class of NEF-PVFs.

The resemblance is easy to prove. First, In both, NEF-PVFs are presented. Second, the Tweedie's article appeared in India (although in a proceeding to a conference also held in Calcutta) while Bar-Lev and Enis' article appeared in The Annals of Statistics (a descendant of The Annals of Mathematical Statistics). Thirdly, the time difference between the two publications is exactly two years. Finally, even though some thirty-five years have passed between the publications of Rao and Blackwell and those of Tweedie and Bar-Lev and Enis, the prevalence of conference proceeding books was still quite rare in western libraries and search engines were still not available. Note that all of the last four points listed are almost identical to those of the case of Rao and Blackwell.

Now, to prove 'independence', I need to present some basic preliminaries of NEFs and their VFs; and then describe the publication process related to Bar-Lev and Enis' (1986) article as well as additional and relevant issues.

\subsection{Some Preliminaries of NEFs, VFs and NEF-PVFs}

I'll first present results from the fundamental paper of Morris (1982) published in The Annals of Statistics in which the VF was used systematically for the first time to handle NEFs. However, a more rigorous approach to Morris' results was given by Letac and Mora (1990). Accordingly, Morris' results are presented in the terminology of that of Letac and Mora (1990).

Let $v$ be a non-degenerate positive Radon measure on $\mathbb{R}$ having a convex support $C$. Let $L: R \rightarrow R$ be its Laplace transform, then the set

$$
D=\theta \in \mathbb{R}: L(\theta)=\int_{\mathbb{R}} e^{\theta x} v(d x)<\infty
$$

is called the effective domain of $v$. Assume that $\Theta \doteq$ int $D$ is nonempty. Then the NEF $F$ generated by $v$ is defined by the set of probabilities

$$
F=F(v)=\{P(v(d x, \theta)=(\exp (\theta x-k(\theta)) v(d x): \theta \in \Theta\},
$$

where $k(\theta) \doteq L(\theta)$, the cumulant transform of $F$, is real analytic and strictly convex on $\Theta$. The $k$-th cumulant of $F$ is the $k$-th derivative of $k(\theta)$. Since $k(\theta)$ is strictly convex on $\Theta$, the mean $m=k^{\prime}(\theta)$ of $P(v(d x, \theta)$ is strictly monotone, and thus its inverse function $\theta=\psi(m)$ is well defined on the open interval $M=k^{\prime}(\Theta)$, where $M$ is called the mean domain of $F$. The variance corresponding to $P\left(v(d x, \theta)\right.$ is $k^{\prime \prime}(\theta)$, or in terms of the mean $m$ is $V(m)=k^{\prime \prime}(\psi(m))$. The pair $(V, M)$ is called the VF of $F$ and it uniquely determines $F$ within the class of NEFs (this result was proved rigorously by Mora (1986)). Also, an NEF $F$ with mean domain $M$ and convex support $C$ is called steep if and only if $M=$ int $C$ (a definition that will be used in the sequel).

Morris (1982) characterized all six quadratic VFs. Letac and Mora (1990) characterized all six cubic VFs. Bar-Lev (1987), in his contribution to the discussion of Jorgensen (1987) paper on exponential dispersion models, showed among a more general setting that any polynomial of the form

$$
V(m)=\sum_{i=1}^{n} a_{i} m^{i}, a_{i} \geq 0, \sum_{i=1}^{n} a_{i}>0,
$$

is a VF with mean domain $M=\mathbb{R}^{+}$of an infinitely divisible NEF. The latter class of polynomial VFs is huge and contains, among others, four out of the six NEFs having up to a quadratic VFs characterized by Morris (1982) and all of the six NEFs having strictly cubic VFs characterized by Letac and Mora (1990).

A large number of papers presenting various types of VFs have appeared in the literature. Many of them are cited in Bar-Lev and Kokonendji (2017). However, it seems obvious that the most interesting VF, which was cited and employed as probability models for dozens of real data sets in numerous areas, is undoubtedly the power VFs having the form

$$
V(m)=a m^{p}, a>0, p \in \mathbb{R} .
$$


A natural question then arises: for which values of $p$ there exists an NEF such that $a m^{p}$ is its VF? Well, a good question, the answer to which actually generates the class of NEF-PVFs.

According to Jorgensen (1987) "a number authors have studied power variance functions, independently, of the present author, for example, Morris (1981), Tweedie (1984), Hougaard (1986), Bar-Lev and of the Enis (1985). The most complete study is by Tweedie (1984), but as shown below, his claim that (2) does not correspond to an exponential family for $p<0$ is not correct". Mr. Tweedie himself wrote in his discussion to Jorgensen's (1987) paper that his paper (Tweedie (1984)) is based on the pre-print of a paper he gave in a conference in Calcutta in 1981. Those conference papers, so it seems, were gathered in 1984 into the Proceedings of the Indian Institute Golden Jubilee International Conference, edited by J. K. Ghosh and J. Roy and published by the Indian Statistical Institute, Calcutta. Also, Mr. Tweedie (perhaps with a dash of cynicism) adds in his discussion that it seems that his paper "seems to have had a wide though informal circulation".

Well, These are the facts I have been able to reveal regarding the 'various' publications of Tweedie on NEF-PVFs. I have, however, two main significant reservations. The first relates to Jorgensen's (1987) statement (already indicated above) that the most complete study of NEF-PVFs was carried out by Tweedie (1984). The second refers to the dates on which Bar-Lev and Enis (1986) results on NEF-PVFs appeared. To provide facts relating to these reservations, I am discomforted forced to tell a little about myself, about my late colleague and friend Peter Enis (who passed away in 2007), our joint work on NEF-PVFs, and the publication dates of the various results. I will do so in the next subsection.

\subsection{Some History and the Notion of Reproducibility of NEFs and NEF-PVFs}

I completed my D.Sc. in May 1981 at the Technion - Israel Institute of Technology. My thesis was on extended exponential families and their applications. After graduating, I joined the Department of Statistics at the University of Haifa in October 1981. Peter Enis was then a member of the Department of Statistics at SUNY Buffalo and served for more than ten years as head of his department. (It should be noted that in the 1970s, this department was one of the leading departments in the US). He spent a sabbatical year in our department in Haifa during the academic year 1981-1982. We quickly became friends and then also research colleagues and started our collaborative research work. The first research issue that concerned us was the reproducibility property which we defined as follows.

Definition 1. Let $F=\left\{P_{\theta}: \theta \in \Theta \subset \mathbb{R}\right\}$ be a family of distributions on $\mathbb{R}$ indexed by a single parameter $\theta$. Let $X_{1}, \ldots, X_{n}$ be i.i.d. r.v.'s with $\mathcal{L}\left(X_{1}\right)=P_{\theta} \in F$ (where $\mathcal{L}(X)$ stands for the law of $X$ ) and set $Y_{n}=\sum_{i=1}^{n} X_{i}$. Then $F$ is said to be reproducible in $\theta$ (or just reproducible) if for all $\theta \in \Theta$ and $n \in \mathbb{N}$, there exists a sequence $(\alpha(n))_{n \geq 1}$ and a mapping $g_{n}: \Theta \rightarrow \Theta$ such that

$$
\mathcal{L}\left(\alpha(n) \sum_{i=1}^{n} X_{i}\right)=P_{g_{n}(\theta)}\left(X_{1}\right) \in F
$$

When investigating this reproducibility property, we focused on NEFs due to their nice analytical features and to my knowledge on the subject. The first case we dealt with was the one with $\alpha(n) \equiv 1$ for all $n$. By inverting (3) in terms of the respective Laplace transforms and solving some functional equations, we showed that for such a case the only reproducible NEF is the Poisson one. This study on the case $\alpha(n) \equiv 1$ was completed in 1982 and was published in Metrika only in 1985 (hereafter, Bar-Lev and Enis (1985a)), rather late, for some editorial reasons which I totally cannot remember what they were. However, during the years 1982 and 1983, we continued our joint research and tried to solve the more general case via functional equations, which again were involved with Laplace transforms. But then Morris' (1982) article on the characterization of NEFs by their VFs was published, so instead of solving the functional equations in terms of Laplace transforms we solved them in terms of the corresponding VFs. Indeed, the equivalent of (3) in terms of VFs is the functional equation

$$
n \alpha^{2}(n) V(m)=V(\alpha(n) m), \text { for all } n \in \mathbb{N}, m \in M,
$$

where $(V, M)$ is the VF the NEF $F$. However, to solve the functional equation (4) we assumed then that, i) $\alpha(n)=n^{\beta}$, where $\beta$ is an arbitrary constant; ii) the mapping $g_{n}: \Theta \rightarrow \Theta$ is onto; and iii) the NEF $F$ is steep, i.e., the mean domain $M$ of $F$ coincides with the interior of its convex support $C$, that is $M=\operatorname{int} C$. Under the latter conditions we proved that the general solution of (4) is indeed of the form (2), i.e., $V(m)=a m^{p}, a>0, p \in \mathbb{R}$. We then proved that the cases $p=0,1,2$ correspond to the normal, Poisson and gamma NEFs, the case $1<p<2$ correspond to compound Poisson NEFs generated by gamma variates, the case $p>2$ corresponds to NEFs generated by positive stable distributions (i.e., with stable index in $(0,1))$, for $0<p<1$ we proved that no NEFs exist and for $p<0$ we proved that no steep NEFs exists (as we got that $M=\mathbb{R}^{+}$is a proper subset of the corresponding convex support $C=\mathbb{R}$ ). These results are similar to those obtained by Tweedie (1984) with one reservation concerning the case $p<0$. For the latter case, we proved that no steep NEFs exist. Tweedie, on the other hand, did not provide any justifiable reason for the nonexistence of NEF-PVFs with $p<0$. Nonetheless, as indicated by Jorgensen (1987), nonsteep NEF-PVFs exist for $p<0$, where the corresponding 
NEFs are generated by absolutely continuous stable distributions with stable index in $(1,2)$ with mean domain $M=\mathbb{R}^{+}$ and convex support $C=\mathbb{R}$ (i.e., $M$ is a proper subset of $C$ and thus the corresponding NEFs are not steep). However, I entirely disagree with Jorgensen, who stated that out of the papers that discussed power VFs (see a list above), Tweedie (1984) carried out the most complete study of NEF-PVFs. Tweedie probably did but also we did. We analyzed NEFPVFs for various properties as infinite divisibility, unimodality, moments, central moments, skewness, general measures of skewness, kurtosis, leptokurticity, upper bounds for (right) tail probabilities and the like.

I will now refer to the publication dates of our results on NEF-PVFs. Our results were first published in a Technical Report No. 89, 1983, published by the Department of Statistics at SUNY Buffalo (hereafter, Bar-Lev and Enis (1983)). The technical report was submitted to The Annals of Statistics and was received there in November 1983. Its last accepted revision was January 1986, and the article was published in 1986. The technical report was also sent to a regular bulk of researchers of the Department of Statistics of SUNY Buffalo. I myself sent it to Lawrence D. Brown who cited it in his research monograph (Brown (1986)) as a Technical Report No. 89, 1984 (instead of 1983). Most interestingly, the paper of Tweedie (1984) did not appear at all in Brown's (1986) list of references. In retrospect and at that point, Peter Enis and I could also write that it seems our technical report "have had a wide though informal circulation" as Tweedie wrote, but we did not.

As far as I can recall now, I received a request from Jorgensen in either late 1986 or early 1987 to contribute a discussion to his paper on exponential dispersion models that was scheduled to appear in the Journal of the Royal Statistical Society, Ser. B., and so I did. As a young academic, I felt that a great deal of respect fell on me when Bent Jorgensen approached me and asked me to contribute my part to the discussion section of his paper (Bent Jorgensen passed away in 2015). Also, as I recall, I sent him a copy of the accepted version of our paper and/or our technical report from 1983, but Jorgensen cited it as Bar-Lev and Enis (1985, a Technical Report). Why he did so and also indicated the year of its publication wrongly, I really do not know and have no clue of it. I guess it was just a kind of oversight.

\section{Why Now? Some Concluding Remarks}

The main question asked here is, why am I raising this issue only now and not before? A good question for which the answer is not so simple and might have different aspects. Here are some:

1. Perhaps that during the mid-1980s and beyond, when I was quite academically young, I felt so honored to contribute a discussion to a significant paper on a new subject (as exponential dispersion models) along with so many wellknown scientists that I had only heard of. Indeed, as cited in Jorgensen's (1987) paper, Tweedie published another paper in 1947 (hereafter, Tweedie (1947)) much before I was even born (Tweedie himself, a physicist, was born in 1919 and passed away in 1996; see link: https://en.wikipedia.org/wiki/Maurice_Tweedie). So maybe I was worried about making an unnecessary fuss which might contain more negative than positive reactions and which might present me as a niggling person. Also, maybe I was worried about my future promotion at my university that might have depended on my scientific relationships with scientists so well known.

2. Now, in retrospect, I was wrong. I had to stand up for credit which undoubtedly Peter Enis an I deserved, without any funk related to my future academic promotion.

3. The inaccurate statement of Jorgensen (1987) that "The most complete study" of NEF-PVFs was presented by Tweedie (1984) has led since 1987 to name the class of NEF-PVFs as the Tweedie class. However, based on all of the findings I presented above, Bar-Lev and Enis contributed to the knowledge of NEF-PVFs at least as much as Tweedie contributed. Consequently, it is entirely evident that both Bar-Lev and Enis should have received the appropriate credit by re-naming the class of NEF-PVFs via the exploitation of the names of Tweedie, Bar-Lev and Enis. This would resemble the dignified and elegant manner Lehmann and Scheffé acted on the Rao-Blackwell Theorem.

4. In my opinion and without any doubt, Lehmann and Scheffé made the right and correct decision by naming the similar results of Rao and Blackwell as the Rao-Blackwell Theorem. As I already indicated, I trust that the two renowned scientists Lehmann and Scheffé, acted correctly and fairly. The late Bent Jorgensen, who published such a fundamental paper on exponential dispersion models, was in charge of the various contributions to the discussion on his paper (after all, he had to respond to each one of them). So he had to realize that our article on the reproducibility property of NEF-PVFs was also "a most complete study of NEF-PVFs," at least as that of Tweedie (1984). But, unfortunately, and whatever be the reason, he had not done so.

5. Now the question is why I bother to write an article so long and with so many details on this issue. Am I doing this for the credit I might get? No, absolutely not, and I hope you do believe in my "absolutely not" response. 
Well, this question has two answers, one major and the other less. The first is a message intended to present and future scientists, not to fear to insist on their mind if they truly believe they are correct and something is wrong (scientifically, of course). Now, one can argue that it is easy for me, in my academic position, to suggest to adopt my present way of thinking and one might be right. Correct, it is easier for me now, but on the other hand even much more difficult. I sincerely regret that in the 1980s, I did not write the present article after the appearance of Jorgensen's (1987) paper and realized that Peter Enis and I did not receive the same credit that Tweedie did, even if such an article would hurt my academic promotion. At this point, I find it most appropriate to quote a line or two from 'if', the Kipling's well-known and stunning poem: "If you can bear to hear the truth you've spoken Twisted by knaves to make a trap for fools,..., Yours is the Earth and everything that's in it, And—which is more-you'll be a Man, my son!”.

My second answer is aimed more at journal Editors, Associate Editors, renowned scientific referees, and the like. Do not underestimate scientific results appearing in new articles submitted for publication by known or unknown scientists (usually young ones), just because their scientific results are inconsistent with your perceptions or conceptions. I want to present an example in this regard. In my role as an Associate Editor for one of the statistical journals, I was asked by the editor to handle a paper that dealt with a Bayesian approach solution to some statistical problem (I myself found the solution to be very innovative and interesting). I sent it to a well-known statistician who replied to me after a week that since he does not believe in Bayesianism to solving statistical problems, he/she will not take the refereeing task of the paper. Just a small example, but a fanatic one.

6. Apropos, while writing this article, I asked one of my most talented Ph.D. students, who is also very knowledgeable in finding rare papers through various search engines, to try and locate Tweedie's (1984) paper. But after a week of searching, he came to my office, all embarrassed and flushed, and told me that he failed to perform the task.

Well, at this point, I trust, the people rest their case.

\section{Acknowledgments}

I am thankful to two anonymous reviewers for helpful comments.

\section{References}

Bar-Lev, S. K. (1987). Discussion on paper by B. Jørgensen, "Exponential dispersion models”. Journal of the Royal Statistical Society Series B, 49, 153-154.

Bar-Lev, S. K., \& Enis, P. (1983). Reproducibility and natural exponential families with power variance functions. Research Report 89, Department of Statistics, State University of New York at Buffalo.

Bar-Lev, S. K., \& Enis, P. (1985). Reproducibility and natural exponential families with power variance functions. Research Report 89, Department of Statistics, State University of New York at Buffalo. https://doi.org/10.1007/BF01897827

Bar-Lev, S. K., \& Enis, P. (1985a). Reproducibility in the one-parameter exponential family. Metrika, 32, 391-394. https://doi.org/10.1007/BF01897827

Bar-Lev, S. K., \& Enis, P. (1986). Reproducibility and natural exponential families with power variance functions. The Annals of Statistics, 14, 1507-1522. https://doi.org/10.1214/aos/1176350173

Bar-Lev, S. K., \& Kokonendji, C. C. (2017). On the mean value parameterization of natural exponential families - a Revisited Review. Mathematical Methods of Statistics, 26(3), 159-175. https://doi.org/10.3103/S1066530717030012

Blackwell, D. (1947). Conditional expectation and unbiased sequential estimation. The Annals of Math. Stat., 18(1), 105-110. https://doi.org/10.1214/aoms/1177730497

Brown, L. D. (1986). Fundamentals of Statistical Exponential Families (with Applications in Statistical Decision Theory). Institute of Mathematical Statistics, Lectures Notes - Monograph Series, V.9, Hayward, California. Retrieved from https://www.jstor.org/stable/4355554.

Hougaard, P. (1986). Survival models for heterogeneous populations derived from stable distributions. Biometrika, 73, 387-396. https://doi.org/10.2307/2336215

Jørgensen, B. (1987). Exponential dispersion models (with discussion). Journal of the Royal Statistical Society, Ser. B, 49, 127-162. https://doi.org/10.1111/j.2517-6161.1987.tb01685.x

Lehmann, E. L., \& Scheffé, H. (1950). Completeness, similar regions, and unbiased estimation. Sankhya, 15, 219-236. 
Letac, G., \& Mora, M. (1990). Natural real exponential families with cubic variance functions. The Annals of Statistics, 18, 1-37. https://doi.org/10.1214/aos/1176347491

Mora, M. (1986). Classification des fontions-variance cubiques des familles exponentielles sur R. C.R. Acad. Sci. Paris 302 Ser. I, 16, 582-591.

Morris, C. N. (1981). Models for positive data with good convolution properties. Memo no. 8949, Rand Corporation, Santa Monica, California.

Morris, C. N. (1982). Natural exponential families with quadratic variance functions. The Annals of Statistics, 10, 65-80. https://doi.org/10.1214/aos/1176345690

Rao, C. R. (1945). Information and accuracy attainable in the estimation of statistical parameters. Bulletin of the Calcutta Mathematical Society, 37, 81-91.

Tweedie, M. C. K. (1947) Functions of a statistical variate with given means, with special reference to Laplacian distributions. Proc. Cambridge Phil. Soc., 49, 41-49. https://doi.org/10.1017/S0305004100023185

Tweedie, M. C. (1984, December). An index which distinguishes between some important exponential families. In Statistics: Applications and new directions: Proc. Indian statistical institute golden Jubilee International conference (Vol. 579, pp. 579-604).

\section{Copyrights}

Copyright for this article is retained by the author(s), with first publication rights granted to the journal.

This is an open-access article distributed under the terms and conditions of the Creative Commons Attribution license (http://creativecommons.org/licenses/by/4.0/). 